\title{
MULTILINE FLOW RATE MEASUREMENT USING ULTRASONIC DOPPLER METHOD
}

\author{
Sanehiro Wada ${ }^{1}$, Hiroshige Kikura ${ }^{1}$, Masanori Aritomi ${ }^{1}$, Yasushi Takeda ${ }^{2}$ \\ and Michitsugu Mori $^{3}$ \\ ${ }^{1}$ Research Laboratory for Nuclear Reactors, Tokyo Institute of Technology, 2-12-1 Ohokayama, Meguro-ku, \\ Tokyo, 152-8550 Japan, e-mail: sane@2phase.nr.titech.ac.jp \\ ${ }^{2}$ Hokkaido University, Kita 8 Nishi 5, Kita-ku, Sapporo 060-0808, Japan \\ ${ }^{3}$ Tokyo Electric Power Company, 4-1 Egasaki-cho, Tsurumi-ku, Yokohama, 230-8510 Japan
}

keywords: Ultrasonic Doppler method, ultrasound, flow rate measurement, velocity profile, non developed flow

\begin{abstract}
Ultrasonic Doppler method for flow metering system has been developed. The principle is based on the integration of instantaneous velocity profile over a pipe diameter so that it is expected to be able to eliminate installation problems such as entry length as well as to follow transient flow rate precisely. Therefore, in the present study, we reported the results of multiline flow rate measurement employing this method Flow metering principle by ultrasonic Doppler method in a circular pipe depends on the alignment of measuring lines. And in most cases, metallic pipes are used both in industrial factories and power plants.

In this paper, the influence of the number of measuring lines and the comparison among several kinds of metallic pipes on the flow rate measurements have been investigated for nondeveloped flows in a vertical pipe.
\end{abstract}

\section{INTRODUCTION}

A flow metering system has been developed using ultrasonic Doppler method [1][2]. This method has many advantages over other flow measurement techniques. One of the advantages is that it has a capability of measuring the velocity profile in a pipe over a diameter, so it is expected to improve the flow metering principle. All conventional flow metering techniques have to assume the velocity profile in a pipe. This assumption limits the improvement of the measurement accuracy just down to only about $2 \%$. In addition, it induces various constraints such as the necessity of installing a long entry length upstream the measurement position. Ultrasonic Doppler method might be able to eliminate these assumption and constraints.

The system has been developed to be applied for conventional piping system of metallic wall [3][4]. Laboratory experiments has shown a very good agreement with the flow rate measurement using conventional orifice flow meter. Additionally, it shows a good tractability for transient behavior. In order to establish the technique and investigate its absolute accuracy, the comparison among experimental results has been performed at the NIST calibration stand [5].

Ultrasonic Doppler method employs a pulsed ultrasonic echography together and detects instantaneous Doppler shift frequency to form a velocity profile. It can obtain spatio-temporal information of the flow field. A pulsed ultrasound is applied also for flow metering based on the traveling time of ultrasonic pulse. It has, however, unfavorable restrictions on its installation and its application is quite limited. Since the ultrasonic Doppler method can obtain an instantaneous velocity profile, it is expected to be applicable for flow metering with much higher accuracy and to be applied with less restrictions. 
Flow metering principle by ultrasonic Doppler method in a circular pipe depends on the alignment of measuring lines. The most accurate way is to make a two or three-dimensional flow mapping at the cross section of the pipe. At one axial location of a cylindrical pipe, the flow rate is estimated by the following equation:

$$
Q(t)=\iint V_{z}(r, \theta, t) r d r d \theta
$$

This requires the measurement of two-dimensional time-dependent velocity distribution. However, it does not require the assumption about any flow conditions such as the degree of flow development, Reynolds number and steadiness. Therefore, it can accomplish a flow metering with minimum restriction and the highest possible accuracy. For the purpose of measuring the flow rate, it also does not require any calibration procedures of the device. However, it is considered that this requires a large number of measuring lines, namely transducers and it takes a long time for data collection. In order to overcome this drawback, we made an assumption that the angular dependence of $V z$ is weak. Adopting this assumption and locating all measuring lines on a diameter (going through the center of the pipe) with inclination angle, reduces the equation to

$$
Q(t)=\sum\left\{\Delta \theta \int V_{z}(r, \theta, t) / \sin (\alpha) r d r\right\}
$$

where $\Delta \theta=2 \mathrm{p} / \mathrm{N}$.

It is expected that this enables one to realize the flow metering with much less number of transducers with reasonably short measuring time. We performed a laboratory experiment in which this simplification is confirmed to be effective using one measuring line. However, it is necessary with regard to the measurement of non-ideal turbulent flows to consider nonaxisymmetric or swirl flows.

In the present study, the influence of the number of measuring lines and the difference of some metallic pipes on the flow rate measurements using ultrasonic Doppler method have been investigated for non-developed flow occurring in a bend pipe.

\section{TRANSMISSION OF ULTRASONIC WAVE}

The propagation and transmission of ultrasound wave follows the common optical law. Reflection, diffraction, and so on occurs at the boundary of two materials which have different acoustic impedance; $Z$. However, when the wall thickness is very small, in the same order of wave length, a sort of resonance transmission of the wave occurs instead at the boundary. We investigated this resonance transmission in this position. When the wall thickness is comparatively small and in the order of the ultrasonic wave length, immersed in a fluid, then the transmission coefficient of the wave, D, is described as follows:

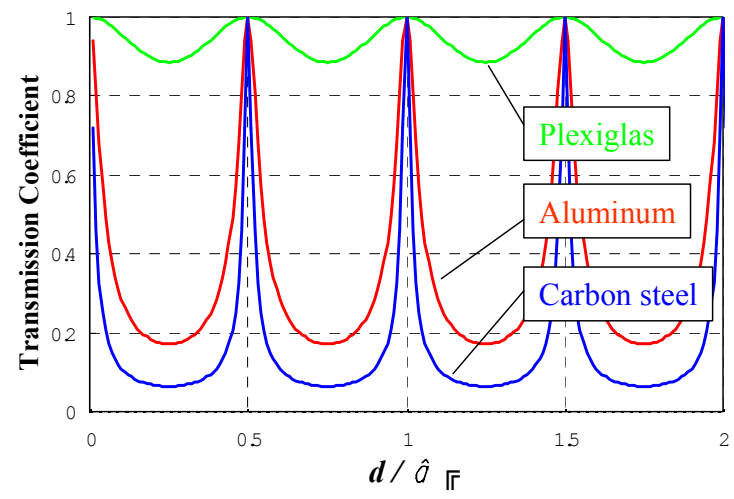

Figure 1. Transmission of ultrasound

$$
D=\left[1+\frac{1}{4}\left(m-\frac{1}{m}\right)^{2} \sin ^{2} \frac{2 \pi d}{\lambda}\right]^{-\frac{1}{2}}
$$


Where $m=Z_{1} / Z_{2}, Z$ is acoustic impedance, $d$ is wall thickness, and $\lambda$ is wave length.

Due to its trigonometric characteristics, it changes periodically as $d / \lambda$. Fig. 1 shows the comparison of the ultrasound transmission in the water for three kind of metallic pipe materials, namely Plexiglas, aluminum and carbon steel. For Plexiglas, the amplitude of oscillation is rather smaller between 80 to $100 \%$ and its width is quite broad. Whereas, for aluminum and carbon steel, the resonance peaks are very strong and very narrow. However, it does promising that for the resonant wall thickness, the transmission is $100 \%$. From the above equation, the maximum transmission occurs at:

$$
d / l=n / 2, \quad(\mathrm{n}=1,2,3 \ldots)
$$

and the minimum (maximum reflection) at:

$$
d / l=(2 n+1) / 4,(\mathrm{n}=1,2,3 \ldots)
$$

\section{EXPERIMENTAL APPARATUS}

A schematic diagram of the experimental apparatus used in this study is shown in Fig.2. The experimental apparatus consists of a water circulation system, a test section and a measurement system. In this study, singlephase turbulent pipe flow in upward direction was investigated.

The experimental apparatus was designed and built in order to put emphasis on the formation of fully developed turbulent pipe flow in both downward and upward directions. The water was circulated by a centrifugal pump from the storage tank into the pipe. The vertical pipe was made of Plexiglas, with the total length, inner diameter and wall thickness were $6 \mathrm{~m}, 50$ $\mathrm{mm}$ and $5 \mathrm{~mm}$, respectively. The flow rate was regulated by the needle valve and monitored by flow orifices and pressure sensor located upstream of the test section.

The test section and the arrangement of ultrasonic (US) transducers are shown in Fig. 3. The water flows into the pipe through the bend pipe and the sudden expansion pipe. The US transducer was mounted on the surface of the outer wall at a contact angle of $\theta$ to the normal plane of the wall. The wall thickness of the pipe in this test section was $5 \mathrm{~mm}$ (Plexiglas), 1.49mm (Carbon steel), $1.61 \mathrm{~mm}$ (Aluminum), because the ultrasonic beam demands low resistance against ultrasonic wave propagation.

Measuring points were located at $0.4 \mathrm{~m}$ (8D), $1.2 \mathrm{~m}$ (24D), $2.4 \mathrm{~m}$ (48D) and 4.8m (96D) downstream from the bend pipe. Three transducers were set on the outer surface of the each measuring point with an interval angle of $120^{\circ}$ and with a constant inclination angle, $\theta=45^{\circ}$

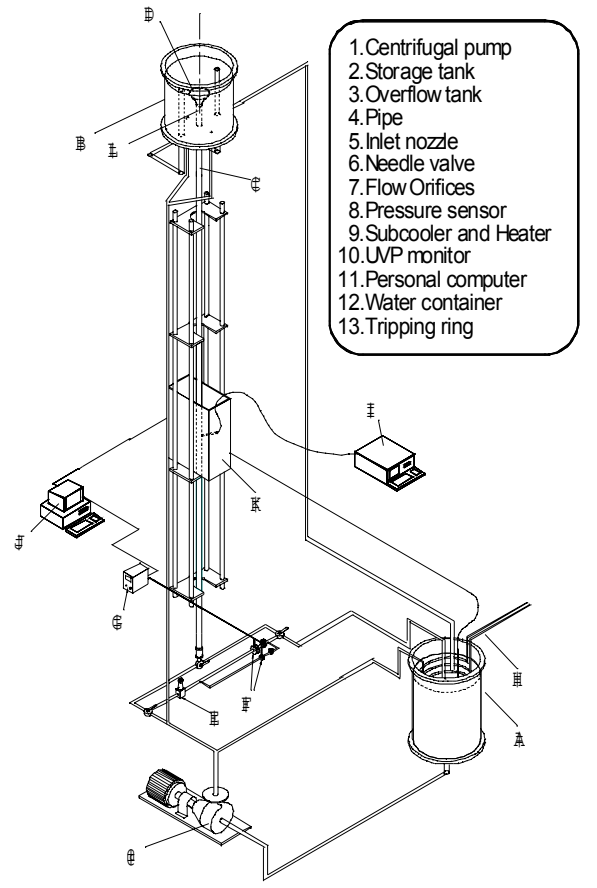

Figure 2. Schematic diagram of the experimental apparatus

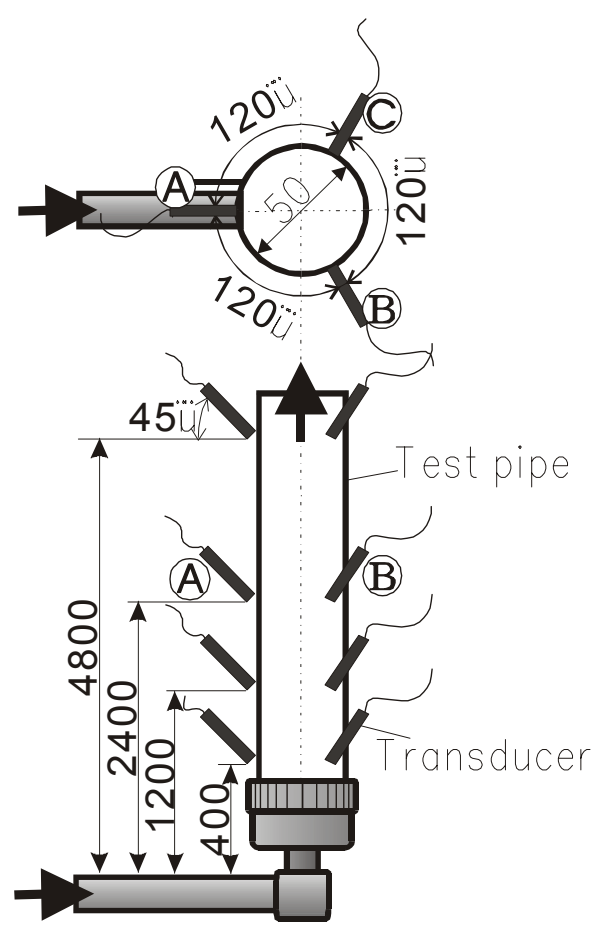

Figure 3. Test section and transducer arrangement in upward direction 
(Plexiglas) and $28.5^{\circ}$ (Carbon steel and Aluminum). These transducers were switched using a multiplexing function of ultrasonic velocity profile monitor. The test section was surrounded by water to form an acoustic coupling between the wall and transducers.

During the experiment, water temperature was kept constant at about $20^{\circ} \mathrm{C}$ using a sub cooler and heater installed inside the storage tank. The flow measurement system consisted of the X3 PS-i model ultrasonic velocity profile monitor (Met Flow AG) and a personal computer, which recorded the flow rate by the flow orifices and temperature data by the thermocouple.

As a reflector material, nylon powder (Daisel Hüls Ltd, WS200P) was suspended in water. The average particle diameter is approximately $80 \mu \mathrm{m}$ and the specific gravity of particle is 1.02 .

\section{MULTI-LINE US TRANSDUCER ARRANGEMENTS}

Fig.4 shows the arrangement of the transducers and measuring lines. At its most simple configuration, ultrasonic Doppler method for flow rate measurement requires only a single transducer whereby the measurement line goes through the center of the pipe. If the flow is axial symmetric, the flow rate can be obtained accurately by integrating the half of the velocity profile using Eq. 4, which is obtained with the measuring line on the diameter as shown in Fig.5.

$$
Q(t)=\frac{\pi}{3}\left\{\frac{R_{0}^{3}-R_{1}^{3}}{R_{0}-R_{0}} v_{0}+\sum_{i=0}^{n-2} \frac{R_{i+1}^{3}-R_{i+2}^{3}}{R_{i+1}-R_{i+2}}\left(v_{i+1}-v_{i}\right)+R_{n}^{2} v_{n}\right\}
$$

For the flow rate calculation, there are two approaches to integrate the velocity profile. For example, using US transducer-A shown in Fig. 4, one method is that the flow rate is calculated by using a measuring line, 1 or 4 , and the others that the flow rate is calculated by using two measuring lines, 1 and 4 . In the same manner, many US transducers can be used to integrate each measuring lines, so that the flow rate can be measured more accurately, even if the flow is non-axisymmetric.

In the present study, we used up to three US transducers for the flow rate calculation.

In the case of using multiline for flow rate calculation, some velocity interpolation of circumferential direction is required as shown in Fig.6, which shows a circumferential

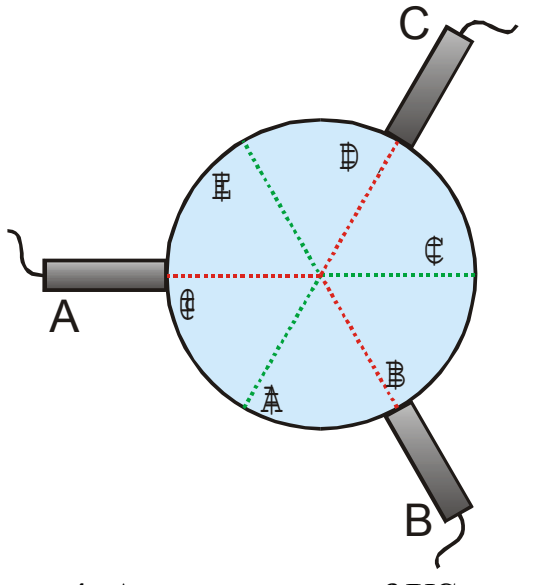

Figure 4. Arrangement of US transducers

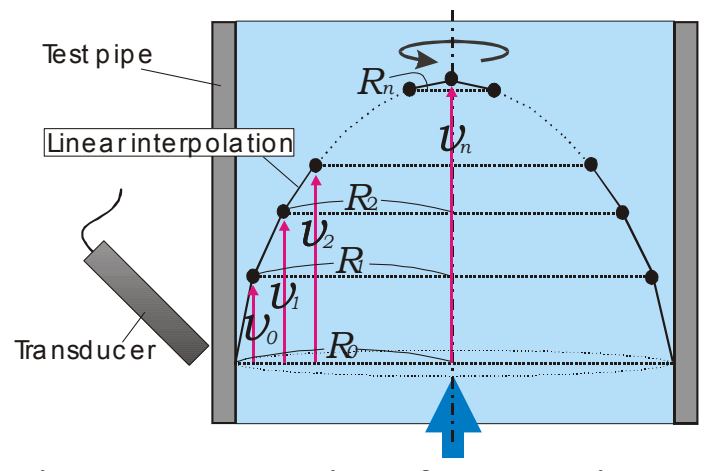

Figure 5. Integration of the velocity profile

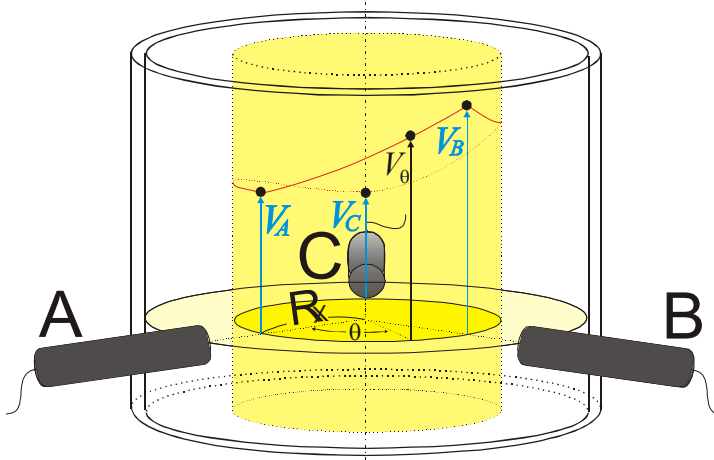

Figure 6. Circumferential velocity interpolation 
velocity interpolation using three measuring lines, 1, 3 and 5 as illustrated in Fig.4. The difference of flow rate error between using Liner and Spline interpolation method was 0.018 $\%$ in this flow, so that we used Liner interpolation for flow rate calculation in this study.

\section{RESULTS AND DISCUSSION}

\subsection{Mean velocity profile}

The mean velocity profiles at the inlet position of $8 \mathrm{D}$ and $96 \mathrm{D}$ from the measurement line using Plexiglas and each US transducer are shown in Fig.7 (a) and (b). The basic frequency of ultrasonic is $4 \mathrm{MHz}$ and Reynolds number is 24000 . From this figure, it is found that the velocity profiles after the bend pipe depends on the inlet length. As shown in Fig.7 (a), for 8D case, the flow is non-axisymmetric so that it is difficult to estimate the flow rate directly using a single US transducer measurement. However, if the flow condition is axial symmetry as shown in Fig.7 (b), for 96D case, the flow might be fully developed, and the flow rate can be calculated by using a half side of the velocity profile.

Fig.7 (c) and (d) show the mean velocity profiles using aluminum and carbon steel at the inlet position of $8 \mathrm{D}$. The half velocity profile that is near side from the transducer is disturbed by a reflection from the front pipe wall, so that it is difficult to directly estimate the flow rate. However, as shown in this figure, the flow rate can be calculated by using the far side profile.

\subsection{Flow rate measurement}

As mentioned above, the velocity profile can be obtained so that a flow rate is estimated by using half region of the velocity profile. Mean flow rate calculated by using multiline US

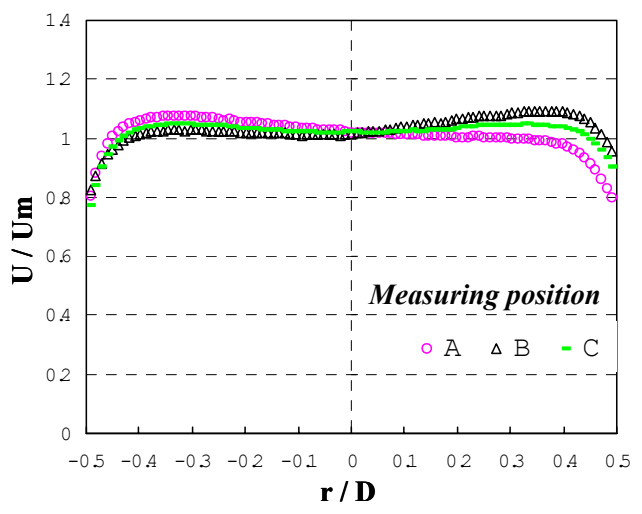

(a) Plexiglas (8D)

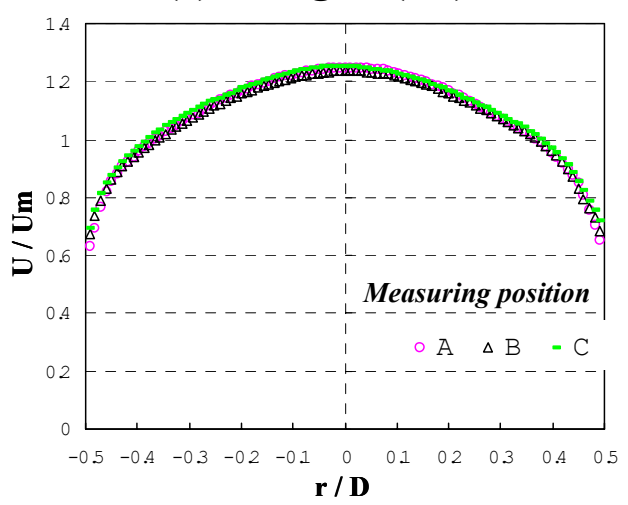

(b) Plexiglas (96D)

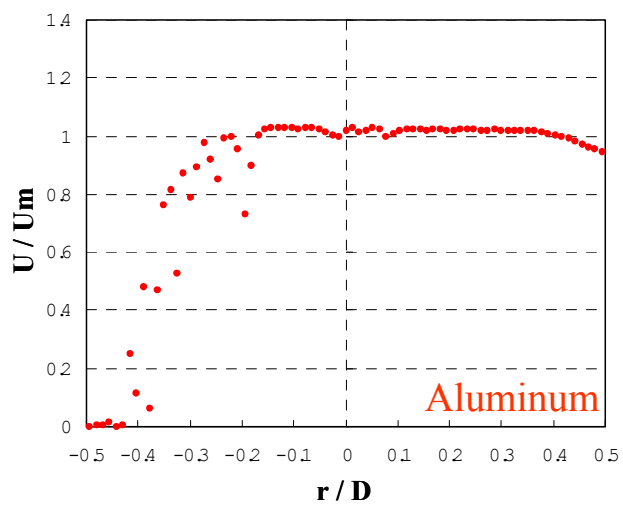

(c) Aluminum (8D)

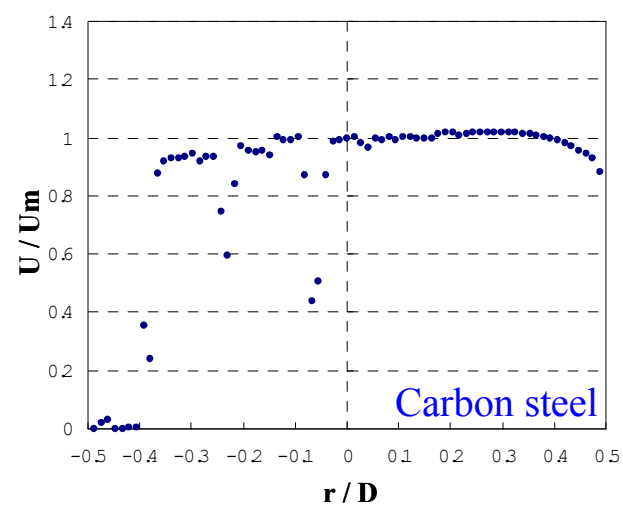

(d) Carbon steel (8D)

Figure 7. Mean velocity profiles 
transducer arrangement is compared with the flow rate measured by the volumetric flow rate. Here the tank capacity is about $1 \mathrm{~m}^{3}$ and the volume of water collected in the tank can be measured with very high accuracy. The volumetric flow rate is estimated from the volume of water collected in the tank and the collection time, so that the expanded uncertainly for this facility is quoted to be $0.08 \%$. Table- 1 shows the comparison of errors between the measured flow rate at $8 \mathrm{D}$ using ultrasonic Doppler method and the volumetric flow rate with respect to the using line number (ref. Fig.4) for calculating a flow rate. As described above, for the metallic wall, it is difficult to estimate a flow rate directly by using the near side velocity profile from the transducer, so that the errors in the case of aluminum and carbon steel are shown only for those cases using the far side profile. And to estimate the accuracy of each measurement, the standard deviation for Plexiglas is plotted in Fig.8. From Table-1 and Fig.8, it is found that for $8 \mathrm{D}$ case, the inlet flow is unstable, so that the standard deviation of the mean flow rate measured by single US transducer is large. However, by using multiple measuring lines, it is achievable to measure the flow rate with high accuracy.

\section{CONCLUSION}

Flow metering system using ultrasonic Doppler method has been developed. In the present study, we reported the results of multiline flow rate measurement employing this method. In this experiment, even for the non-axisymmetric flow occurred at the inlet position through the pipe bend and sudden expansion pipe, it is possible to measure the flow rate using multiline system with high accuracy.

As mentioned above, it is indicated that the present multiline flow metering system by using ultrasonic Doppler method has very high accuracy. And this method can be considered as a multipurpose system, because the flow rate can be measured under non-ideal flow conditions such as non-developed flow or non-axisymmetric flow.

Table 1. Comparison of errors for each material

\begin{tabular}{|l|c|c|c|}
\hline $\begin{array}{l}\text { Using } \\
\text { line } \\
\text { number }\end{array}$ & \multicolumn{3}{|c|}{ Error \% } \\
\cline { 2 - 4 } & Plexiglas & Aluminum & $\begin{array}{c}\text { Carbon } \\
\text { steel }\end{array}$ \\
\hline 1 & 1.99 & - & - \\
\hline 2 & 0.55 & -3.7 & 0.03 \\
\hline 3 & -2.33 & - & - \\
\hline 4 & -5.63 & 1.51 & -3.14 \\
\hline 5 & -0.95 & - & - \\
\hline 6 & 4.09 & -1.41 & -3.18 \\
\hline 1,4 & -1.68 & - & - \\
\hline 3,6 & 0.98 & - & - \\
\hline 2,5 & -0.20 & - & - \\
\hline $1,3,5$ & -0.40 & - & - \\
\hline $2,4,6$ & -0.17 & -1.15 & -2.07 \\
\hline $1-6$ & -0.28 & - & - \\
\hline
\end{tabular}

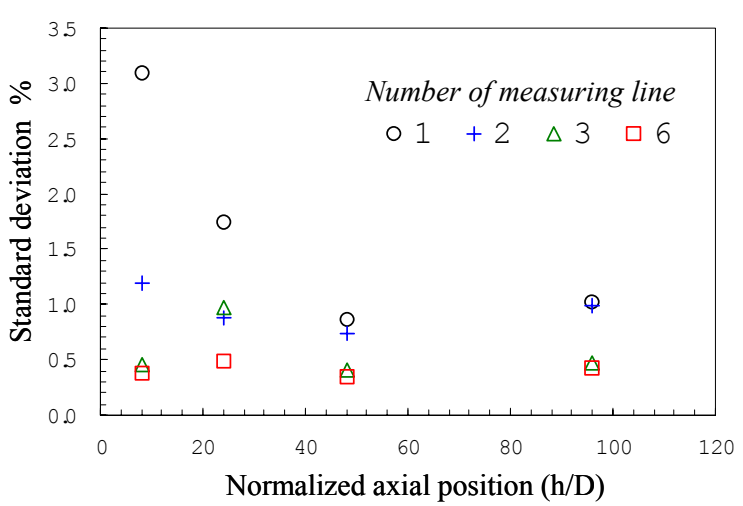

Figure 8. Standard deviation (Plexiglas) 


\section{REFERENCES}

[1] Takeda, Y., Measurement of velocity profile of mercury flow by ultrasound Doppler shift method, Nuclear Technology, 79 (1987), pp 120-124.

[2] Takeda, Y., Velocity profile measurement by ultrasonic Doppler method, Experimental Thermal and Fluid Sci., 10 (1995), pp 444-453.

[3] Kikura, H., et al., Proc. of $3^{\text {rd }}$ ASME/JSME Joint Fluid Engin. Conf. (1999), FEDSM997141.

[4] Mori, Metallic., 7th Int. Conf. on Ncl. Engin. (ICONE7), Tokyo (1999), ICONE-7429.

[5] Takeda, Y., et al., ASME 2000 Fluids Engn. Divi. Summer Meet.,(2000), FEDSM200011102 